\title{
Near-Ideal Strength in Gold Nanowires Achieved through Microstructural Design
}

\author{
Chuang Deng and Frederic Sansoz* \\ School of Engineering and Materials Science Program, The University of Vermont, Burlington, Vermont 05405
}

M icrostructural design has been exploited for some time to enhance the strength of crystalline materials, mostly through work-hardening to increase the initial density of dislocations, ${ }^{1}$ or grain size refinement in the microcrystalline and nanocrystalline regimes following the classical Hall-Petch relation., ${ }^{2,3}$ More recently, it was also observed that bulk specimens of ultrafine-grained and nanocrystalline Cu possess much higher yield strength when coherent twin boundaries (CTBs) are introduced during electrochemical deposition. ${ }^{4-6}$ Crystalline materials, however, cannot be strengthened unlimitedly by decreasing the mean grain size or twin size. For instance, a reverse Hall-Petch effect, which is characterized by a strength reduction as the grain size is decreased below $10-20 \mathrm{~nm}$, has been found in $\mathrm{Cu}^{7}$ and other face-centered cubic (FCC) nanocrystalline metals; ${ }^{8}$ here a transition in deformation mechanism from dislocation-mediated plasticity to grainboundary sliding below a critical grain size was found responsible for this phenomenon. Similarly, a strongest twin size was discovered in nanotwinned $\mathrm{Cu}$, which originates from a shift in yielding mechanism from slip transfer across twin boundaries to easy dislocation nucleation from atomic ledges existing in twin boundaries. ${ }^{5}$

In addition to microstructure effects, modifications of external geometry and surface structure can strongly influence strength in crystalline samples with nanometer sizes. Particularly, significant sample size dependence has been observed experimentally on the strength and crystal plasticity of various FCC metals such as $\mathrm{Ni}^{9,},{ }^{10} \mathrm{Ag}^{11}$ and $\mathrm{Au}_{1}{ }^{12-15}$ which has led to the conclusion that smaller is stronger. It should be emphawww.acsnano.org
ABSTRACT The ideal strength of crystalline solids refers to the stress at elastic instability of a hypothetical defect-free crystal with infinite dimensions subjected to an increasing load. Experimentally observed metallic wires of a few tens of nanometers in diameter usually yield far before the ideal strength, because different types of surface or structural defects, such as surface inhomogeneities or grain boundaries, act to decrease the stress required for dislocation nucleation and irreversible deformation. In this study, however, we report on atomistic simulations of near-ideal strength in pure Au nanowires with complex faceted structures related to realistic nanowires. The microstructure dependence of tensile strength in face-centered cubic Au nanowires with either cylindrical or faceted surface morphologies was studied by classical molecular dynamics simulations. We demonstrate that maximum strength and steep size effects from the twin boundary spacing are best achieved in zigzag Au nanowires made of a parallel arrangement of coherent twin boundaries along the axis, and $\{111\}$ surface facets. Surface faceting in Au NWs gives rise to a novel yielding mechanism associated with the nucleation and propagation of full dislocations along \{001\}<110 slip systems, instead of the common $\{111\}\langle 112\rangle$ partial slip observed in face-centered cubic metals. Furthermore, a shift from surface dislocation nucleation to homogeneous dislocation nucleation arises as the twin boundary spacing is decreased below a critical limit in faceted nanowires. It is thus discovered that special defects can be utilized to approach the ideal strength of gold in nanowires by microstructural design.

KEYWORDS: gold nanowire $\cdot$ faceting $\cdot$ twin boundary $\cdot$ dislocation $\cdot$ molecular dynamics

sized however that the internal microstructure still plays a key role in the mechanical behavior of nanosized crystalline materials. For example, Wu et al. ${ }^{12}$ have directly measured with atomic force microscopy-based bending experiments that Au nanowires (NWs) with a diameter of $40 \mathrm{~nm}$ could be up to 100 times stronger than conventional bulk Au. These authors have also found that the yield strength of Au NWs grown by electrodeposition technique was significantly lowered after thermal annealing, which indicated that certain internal defects or microstructures could be responsible for the observed increase in strength with decreasing NW diameter. Maa $\beta$ et al. 16,17 have also argued that the initial microstructures of metal nanopillars similar in size and shape to those tested by
*Address correspondence to frederic.sansoz@uvm.edu.

Received for review June 24, 2009 and accepted September 3, 2009.

Published online September 10, 2009.

10.1021/nn900668p CCC: \$40.75

() 2009 American Chemical Society 
others, ${ }^{10,13,14}$ including grain boundaries and surface dislocations added during microfabrication, could contribute to the apparent increase in strength.

Recent advances in materials synthesis have enabled making FCC metal NWs with a variety of microstructures and surface morphologies. For example, past transmission electron microscopy (TEM) studies have shown the existence of bamboo-like structures in $\mathrm{Au}$ and $\mathrm{Cu}$ NWs grown by electrodeposition. ${ }^{18,19}$ Such structures consist of a parallel arrangement of planar defects perpendicular to the wire axis, which correspond to (111) CTBs. Also, single-crystalline and nanocrystalline microstructures in Au NWs were created by electrodeposition using different applied overpotentials. ${ }^{18}$ Furthermore, ultrathin [111] oriented Au NWs with diameters less than $10 \mathrm{~nm}$ have been synthesized by reduction of $\mathrm{HAuCl}_{4}$ in oleic acid and oleylamine. ${ }^{20,21}$ Halder and Ravishankar ${ }^{22}$ have developed a bottom-up approach to produce ultrafine Au NWs by an oriented attachment of Au nanoparticles. Different TEM studies $^{22-26}$ have shown that aligned Au nanoparticles can fuse together at $\{111\}$ facets to form faceted $\mathrm{Au}$ NWs, during which twins can be easily formed in favor of energy reduction. It is worth noting that although most facets were found to be $\{111\}$ planes in Au nanoparticles, $\{100\}$ facets have also been frequently observed. $22,23,26$ Other FCC metal NWs, such as $\mathrm{Cu}$ and Ag NWs, also tend to have $\{111\}$ and $\{100\}$ nanoscale facets. ${ }^{24,26,27}$ For example, faceted twinned structures, which usually exhibited a zigzag shape, have been observed in Cu nanorods grown by magnetron sputter deposition. ${ }^{27}$ Furthermore, $\{111\}$ surface facets are often encountered in twinned semiconducting NWs grown by the vapor-liquid-solid process ${ }^{28-30}$ and standard commercial metal organic vapor phase epitaxy. ${ }^{31}$ Shim et al. ${ }^{32}$ have proposed a model based on the energy minimization of interfaces and edges near twin boundaries to account for the $\{111\}$ faceted-twinning structure in semiconducting NWs.

Molecular dynamics (MD) simulations have been used in the past to characterize the influence of microstructure on the elastic and plastic properties of nanosized FCC metals. Monk and Farkas ${ }^{33}$ have predicted that dislocation-mediated plasticity decreases with decreasing wire diameter in nanocrystalline Ni NWs. Cao et al., ${ }^{34}$ who have simulated the tensile behavior of both single-crystal and nanocrystalline Cu NWs, found that the yield strength in the latter was significantly smaller as a result of dislocation emission from grain boundaries instead of free surfaces. Moreover, periodically twinned circular $\mathrm{Au}^{35,36}$ and $\mathrm{Cu}^{37} \mathrm{NWs}$ containing parallel (111) CTBs have been studied by MD simulations, where it was shown that the intersection of CTBs with the free surface in NWs acts as preferential sites for the nucleation and propagation of dislocations during plastic deformation. Furthermore, it has been predicted by atomistic simulation that external changes of surface morphology with different faceted structures can dramatically influence the yield strength of stable $\langle 110\rangle$ oriented $\mathrm{Ag} \mathrm{NWs}^{38}$ and $\mathrm{Cu} \mathrm{NWs}{ }^{39}$ deformed in tension.

This work poses a fundamental question: can defects, both internal (e.g., grain boundaries) and external (e.g., surface facets), give rise to ideal strength in realistic metal NWs through microstructural design? In the present study, MD simulations were performed to investigate the tensile behavior of Au NWs containing different types of microstructure observed experimentally. Here, we discover that twin defects can be utilized to approach the ideal strength of gold in faceted NWs, whose elastic limit is dramatically larger than that found in single-crystalline and polycrystalline Au NWs with perfectly circular cross-section. This theoretical study sheds new light on the plasticity and mechanical strength of faceted Au NWs at extremely small scale and paves the way for designing more robust nanostructures.

\section{RESULTS AND DISCUSSION}

Tensile Strength of Perfectly Cylindrical Au NWs. Figure 1 shows the atomic structure and tensile stress - strain curve of cylindrical Au NWs with a circular cross-section made of different types of microstructure. Four classes of circular NWs are represented in this figure: polycrystalline Au NWs (Figure 1a), polycrystalline Au NWs with grains containing nanoscale twins of $2 \mathrm{~nm}$ in size (Figure 1b), twinned Au NWs with a periodic arrangement of parallel (111) CTBs, similar to the bamboo-like structure described above (Figure 1c), and the same twinned Au NWs containing also one atomic ledge per boundary, as this type of defect has been observed in nanotwinned $\mathrm{Cu}^{5}$ (Figure 1d). The mean grain size of the polycrystalline Au NWs in Figure $1 \mathrm{a}, \mathrm{b}$ is $16 \mathrm{~nm}$. The twin boundary spacing (TBS) in the bamboo-like Au NWs presented in Figure $1 c, d$ is $8 \mathrm{~nm}$. For comparison, the stress - strain curve for a defect-free single-crystal $\mathrm{Au}$ NW is represented in this figure by a dashed line. The sample diameter and periodic length are 20 and $32 \mathrm{~nm}$, respectively, for all NWs.

Figure 1 parts $\mathrm{a}$ and $\mathrm{b}$ show that the maximum flow strength of nanocrystalline Au NWs under tension is significantly smaller than that in single-crystal Au NWs with same diameter. This result is in good agreement with previous atomistic predictions in nanocrystalline $\mathrm{Ni}^{33}$ and $\mathrm{Cu}^{34} \mathrm{NWs}$, where it was shown that grain boundaries are the main sources of dislocations during plastic deformation. In contrast, single-crystalline Au NWs have been found to yield by nucleating $1 / 6\{11 \overline{1}\}\langle 112\rangle$ partial dislocations from the free surface, ${ }^{40}$ which requires larger stress than the nucleation of partial dislocations from grain boundaries. ${ }^{34}$ Also, it is worth noting that nanocrystalline Au NWs with grains containing nanoscale twins (Figure 1b) exhibit higher strength than those with no twins (Figure 1a), which confirms earlier re- 
ports from experiments ${ }^{4,5}$ and MD simulations ${ }^{41}$ about the strengthening effects of twin boundaries in nanocrystalline materials. Moreover, Figure 1a,b shows that the Young's modulus of polycrystalline Au NWs is significantly lower (by about half) than that of single-crystalline NWs ( $130 \mathrm{GPa}$ ). This finding is consistent with previous studies ${ }^{35}$ showing that the Young's modulus of [111] oriented singlecrystalline Au NWs with diameters ranging from $5-10 \mathrm{~nm}$ remains constant at $124 \mathrm{GPa}$, which is about $60 \%$ higher than that observed in bulk polycrystalline $\mathrm{Au}(78 \mathrm{GPa})$. This observation is also supported by the fact that grain boundaries cause some significant microplasticity (e.g., grain boundary sliding) early in the deformation of nanocrystalline $\mathrm{Au}$ NWs, which significantly decreases their elastic limit under tensile loading. Furthermore, the flow stress of polycrystalline $\mathrm{Au}$ NWs with twins inside each grain is notably larger than that without twins, as shown in Figure 1b, because twin boundaries can effectively suppress grain boundary-induced microplasticity in nanocrystalline metals. ${ }^{41}$

The behavior of perfectly circular Au NWs with nanoscale twins shown in Figure 1c,d appears to be notably different from that of nanocrystalline NWs described above. The tensile stress - strain curve in Figure 1c reveals that the yield stress is lower and the maximum flow stress higher in twinned Au NWs, as compared with the yield strength of the single-crystalline NW. This result is consistent with an earlier report ${ }^{36}$ where we showed that CTBs can be strong barriers to the glide of partial dis-

locations in $\mathrm{Au}$; therefore causing dramatic increase of plastic flow stress in Au NWs with nanoscale twins due to complex dislocation-CTB interactions. Past studies have also shown that strong size effects exist in both the yield stress ${ }^{42}$ and maximum flow stress $^{36}$ of cylindrical Au NWs with nanoscale twins;

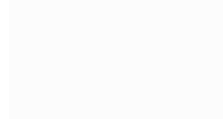

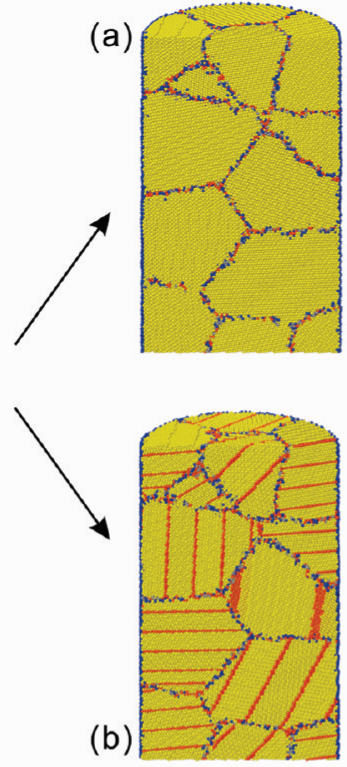
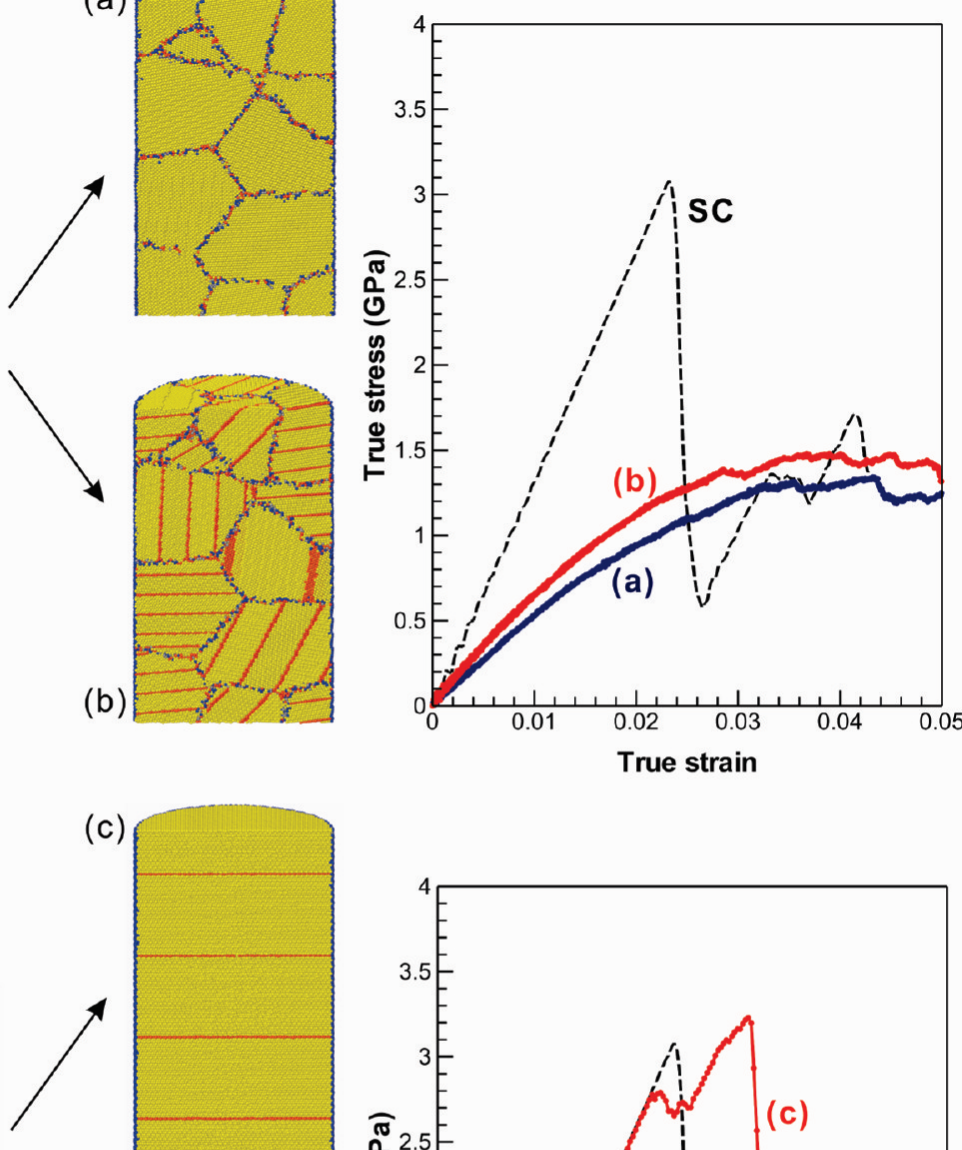

(d)
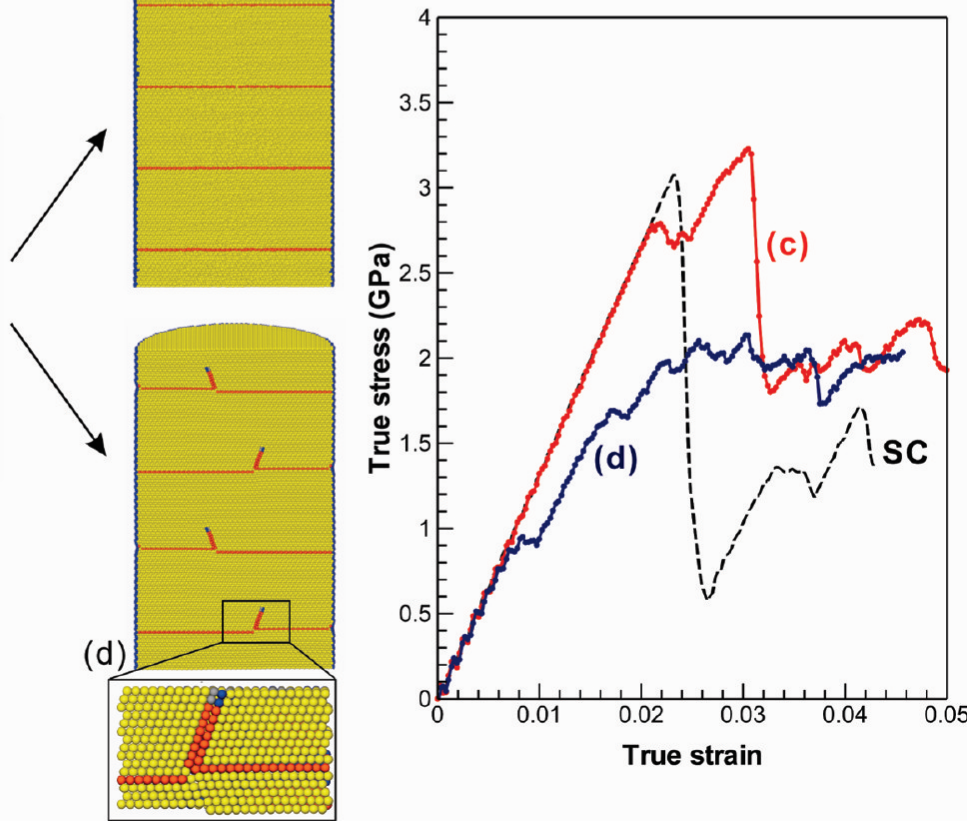

Figure 1. Influence of microstructure on the mechanical behavior of pure Au nanowires with circular cross-section deformed in tension by molecular dynamics simulations. Randomly oriented polycrystalline nanowires with a mean grain size of $16 \mathrm{~nm}$ whose grains contain either (a) no lattice defects or (b) intrinsic stacking faults. [111] oriented twinned nanowires with bamboo-like structure containing a periodic arrangement of (c) (111) coherent twin boundaries or (d) twin boundaries with one atomic ledge per boundary. The close-up view in (d) shows the atomic details of a ledge on the twin plane. The dashed line shown for comparison represents the simulated stress-strain curve of a single crystalline (SC) Au nanowire in the absence of defects. The diameter and periodic length of all nanowires are equal to 20 and $32 \mathrm{~nm}$, respectively. The atoms are colored according to Ackland and Jones' crystal structure analysis. ${ }^{51}$ cylindrical twinned Au NWs can be either stronger or weaker than their single-crystalline, twin-free counterparts with identical diameter, depending on both TBS and NW diameter. Furthermore, Figure 1d shows that the maximum strength of cylindrical twinned Au NWs decreases markedly by adding one 


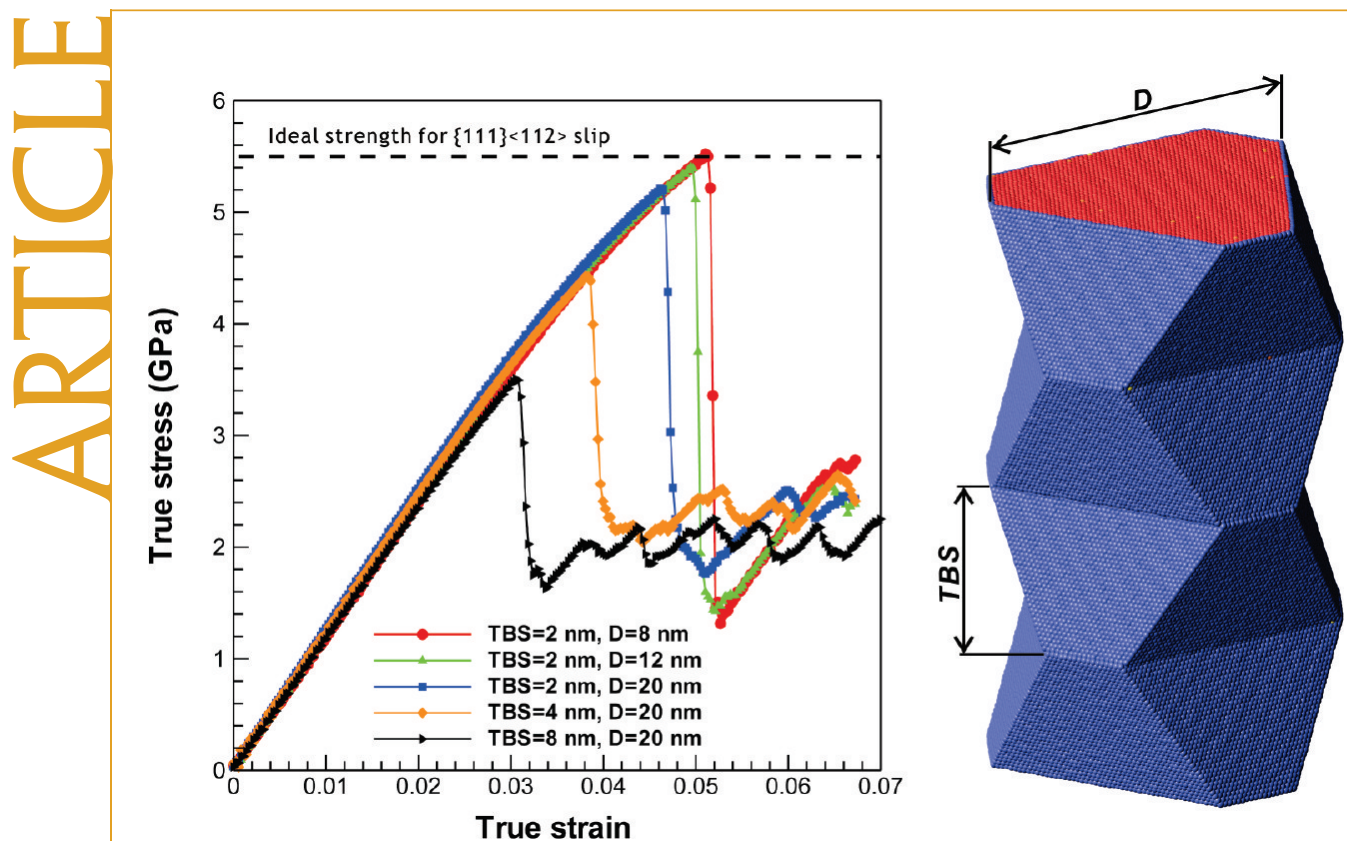

Figure 2. Tensile stress - strain curves of [111] oriented Au nanowires with $\{11 \overline{1}\}$ surface facets and parallel distribution of (111) coherent twin boundaries for different diameter $(D)$ and twin boundary spacing (TBS). The horizontal dashed line marks the ideal strength required for nucleation of $\{111\}\langle 112\rangle$ slip in an infinite, defect-free Au crystal loaded in tension along the [111] direction. The atomistic model of a faceted Au nanowire with $D=20 \mathrm{~nm}$ and TBS $=8 \mathrm{~nm}$ is shown in the righthand side. The periodic length is $32 \mathrm{~nm}$ for all nanowires.

atomic ledge to each twin boundary. This behavior could stem from two reasons. First, each atomic ledge, formed here by a shift of a portion of the twin boundary along the [111] direction, dissociates into an extended stacking fault after relaxation (see inset in Figure 1d). During tensile deformation, the partial dislocations from such extended stacking faults move easily away from the twin boundary and thus act to decrease the yielding stress in the NW. Second, as the deformation continues, pre-existing atomic ledges become source of new partial dislocations, which in turn form full $\{111\}\langle 110\rangle$ dislocations by recombining with partial dislocations previously emitted from the CTBs. The newly formed full dislocations are able to penetrate the $\mathrm{CTBs},{ }^{38}$ which may also cause the Au NW to fail at a smaller stress than that in twinned Au NWs containing no atomic ledges. It should be mentioned however that the Young's modulus in cylindrical twinned Au NWs is almost identical to that in single-crystalline Au NWs $(\sim 130 \mathrm{GPa})$ which is not significantly affected by the NW diameter, ${ }^{36}$ twin boundary spacing, ${ }^{36}$ or existence of atomic ledges.

In summary, it can be concluded that the strongest microstructure in cylindrical Au NWs with a diameter of $20 \mathrm{~nm}$ corresponds to a bamboo-like microstructure with parallel (111) CTBs, which here was found to exhibit a maximum strength of $3.23 \mathrm{GPa}$, that is, slightly larger than the tensile strength of a single-crystalline NW in the absence of defects (3.08 GPa).

Tensile Strength of Faceted Au NWs. \{111\}-Type surface facets have been widely observed in twinning superlattices in semiconducting NWs, ${ }^{28-32}$ as well as in zigzag twinning structures in FCC metal NWs. ${ }^{22,25,27}$ It is well-established that $\{111\}$ planes are preferential close-packed planes for the easy glide of dislocations in FCC metals. ${ }^{5,10,13,16}$ However, a special feature of zigzag FCC NWs with $\{11 \overline{1}\}$ facets is that all $\{11 \overline{1}\}$ slip planes are parallel to a facet of the free surface. It should be mentioned that such characteristics do not occur with other types of facets. ${ }^{38,39}$ Therefore the yielding mechanism of Au NWs with $\{11 \overline{1}\}$ facets becomes profoundly different from that in the cylindrical NWs with circular crosssection, as shown below.

Figure 2 shows the atomic structure and tensile stress-strain curve for different [111] oriented Au NWs with $\{11 \overline{1}\}$ surface facets, where $D$ and TBS represent the diameter and twin boundary spacing, respectively, as defined in the atomistic model shown in this figure. Each curve presents an elastic regime up to 3-5\% strain, followed by a sharp yield point and an abrupt drop in tensile stress, corresponding to the nucleation and propagation of the very first dislocations in the crystal lattice. It is important to note that faceted $\mathrm{Au}$ NWs are much stronger in comparison to cylindrical $\mathrm{Au}$ NWs shown in Figure 1. For example, under the same simulation conditions, the lowest tensile strength calculated in faceted Au NWs is equal to $3.5 \mathrm{GPa}$ for a faceted NW with $D=20 \mathrm{~nm}$ and TBS $=8 \mathrm{~nm}$, which is already higher than the maximum flow stress calculated in all cylindrical Au NWs under tensile deformation (3.23 GPa). It can also be noted in Figure 2 that the yield strength of faceted Au NWs increases as both $D$ and TBS decreases, but such variations are clearly more significant when TBS varies. As such, this figure reveals that the strength of faceted Au NWs with TBS $=2 \mathrm{~nm}$ and a small diameter such as $D=8 \mathrm{~nm}$ is near the ideal strength of $\mathrm{Au}$, which is on the order of $5.5 \mathrm{GPa}$ based on our calculations. ${ }^{43}$

Diao et al. ${ }^{44}$ have also shown by atomistic simulation that the free surface of [111] oriented Au NWs in tension causes significant compressive stress in the interior of the specimen. Therefore, the smaller the NW diameter, the larger the internal compressive stress, and the larger the external stress required to yield the NW. This idea also supports the strength increase as a function of the diameter as observed in Figure 2 for faceted NWs with TBS $=2 \mathrm{~nm}$.

Furthermore, we represent in Figure 3 the evolution of the calculated yield strength in faceted Au NWs 
as a function of the number of twin boundaries per unit length, 1/TBS. This figure shows that the yield strength varies from 3.5 to $5.27 \mathrm{GPa}$ with decreasing TBS from 8 to $1.4 \mathrm{~nm}$, respectively, which corresponds to a $50 \%$ increase in strength. Another peculiar behavior is that there is a clear shift in plastic behavior for TBS $=2.6 \mathrm{~nm}$ or $1 / \mathrm{TBS}=0.38$, as the yield strength approaches the ideal strength for $\mathrm{Au}$, suggesting a transition in the underlying mode of deformation, as described hereafter.

Mechanisms of Incipient Plasticity in Faceted Au NWs. To understand the ultrahigh strength of faceted Au NWs with nanoscale twins, and its steep size dependence as a function of 1/TBS, we performed an atomic-scale analysis of deformation mechanisms in each NW at yield point. As mentioned above, the mechanism of surface dislocation nucleation on $\{111\}$ planes ${ }^{12,15,36-38,40}$ is predicted to be difficult in Au NWs with $\{11 \overline{1}\}$ facets. However, it is shown in Figure $4 a$ that the yielding of a faceted Au NW with $D=20 \mathrm{~nm}$ and TBS $=8 \mathrm{~nm}$ (yield strength $=3.5 \mathrm{GPa}$ ) occurs by the nucleation and propagation of full (001) [110] dislocations, instead of the common $\{11 \overline{1}\}\langle 112\rangle$ partial slip observed in FCC metals, which may account for the enhanced strength of faceted NWs relative to circular ones. This process of dislocation emission is surface-mediated and sitespecific, since it occurs at the intersection of CTBs with the free surface, which is similar to the nucleation of partial dislocations in perfectly circular twinned $\mathrm{Au}$ $\mathrm{NWs}{ }^{37}$ It has been reported in the literature that full lattice dislocations in FCC metal NWs deformed under uniaxial loading could be transmitted through (111) CTBs by $\{001\}\langle 110\rangle$ slip from the parent grain to the twin grain. ${ }^{36,45}$ To our knowledge, however, the direct emission of $\{001\}\langle 110\rangle$ dislocations from free surfaces has never been reported in either bulk or nanosized FCC metals. In the present study, this mechanism is made possible in [111] oriented Au NWs because the corresponding Schmid factor is very high (0.41) for this slip system under uniaxial tension.

The strong size dependence of yield strength in faceted Au NWs with decreasing TBS shown in Figures 2 and 3 can be interpreted from the coupled effects of CTBs and free surfaces on the necessary stress to emit and propagate a dislocation as follows. Chen et al. ${ }^{46}$ have shown that CTBs exert a strong repulsive image force on dislocations that move toward the boundary. According to this model, the image shear stress due to the closest CTB that opposes to the glide of full screw dislocations, $\tau_{\mathrm{ctb}}$, is such that ${ }^{46}$

$$
\tau_{\mathrm{ctb}}=-\frac{\lambda G b}{4 \pi L_{\mathrm{B}}}
$$

where $L_{B}$ is the distance between the dislocation and the closest CTB, $\lambda$ is a dimensionless measure of the intrinsic strength of the CTB due to elasticity mismatch between parent and twin grains at the twin interface, ${ }^{46}$

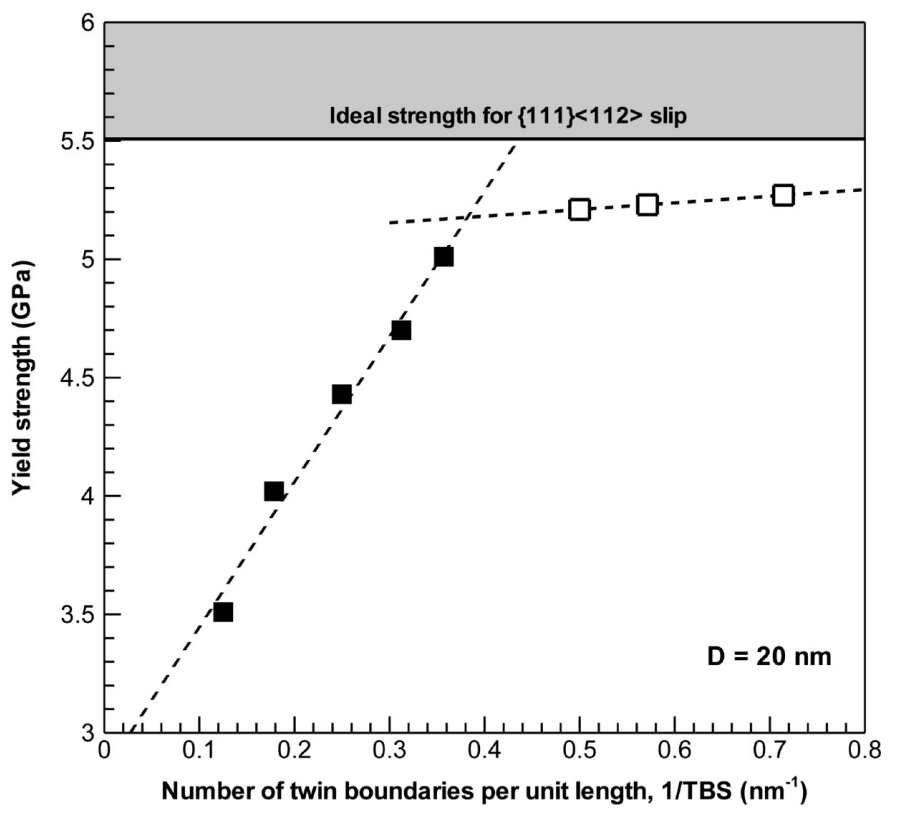

Figure 3. Evolution of yield strength as a function of number of twin boundaries per unit length in $20-\mathrm{nm}$-diameter Au nanowires with $\{111\}$ surface facets. A shift in yielding mechanism from surface dislocation nucleation (filled symbols) to homogeneous dislocation nucleation (open symbols) appears clearly for TBS $=2.6 \mathrm{~nm}$ or $1 /$ TBS $=0.38 \mathrm{~nm}^{-1}$.

$G$ is the shear modulus, and $b$ is the magnitude of the Burgers vector. In the present study, the characteristic length $L_{B}$ is always equal to TBS/sin $\theta$, with $\theta$ being the angle formed by the slip plane with the twin boundary, since all full dislocations are nucleated from the intersection of CTBs with the free surface. Consequently, the image force imposed by CTBs on the emission of new dislocations must be added to the applied stress such as

$$
\tau_{\mathrm{ctb}} \propto \frac{1}{\mathrm{TBS}}
$$

Figure 3 enables us to validate eq 2 on the basis that a linear dependence between yield strength and number of twin boundaries (per unit length) is clearly evident from our atomistic predictions for TBS larger than or equal to $2.6 \mathrm{~nm}$, that is, 1/TBS being smaller than or equal to 0.38 .

Furthermore, we found that the stress plateau shown in Figure 3 for 1/TBS larger than 0.38 corresponds to a shift from site-specific surface dislocation nucleation to homogeneous dislocation nucleation. For example, Figure $4 \mathrm{~b}$ proves that the yielding of Au NWs with $D=8 \mathrm{~nm}$ and TBS $=2 \mathrm{~nm}$ occurs by homogeneous nucleation of $\{11 \overline{1}\}\langle 112\rangle$ partial dislocations inside the NW, as opposed to the surface nucleation of full $\{001\}\langle 110\rangle$ dislocations in Au NWs with TBS $=8 \mathrm{~nm}$ as shown in Figure $4 a$. The dynamic process of homogeneous dislocation nucleation can also be found in the movie given in the (Supporting Information). The transition in yielding mechanism for faceted Au NWs subjected to tensile deformation can therefore be accounted for by the excessive increase in image force imposed by CTBs as TBS is decreased below a critical 
(a)

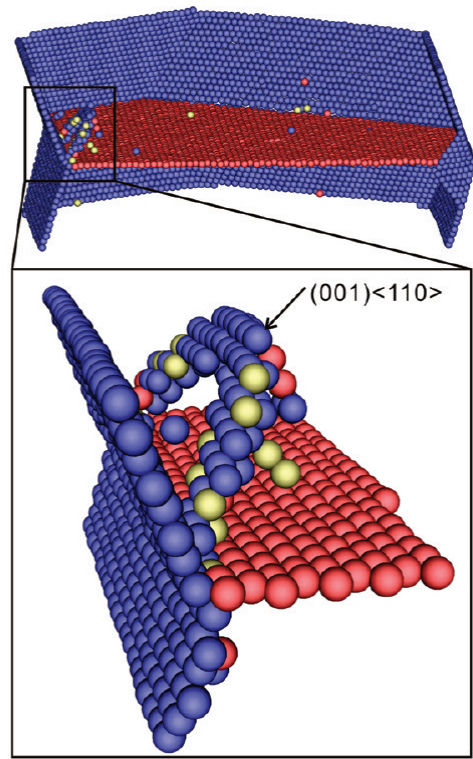

(b)

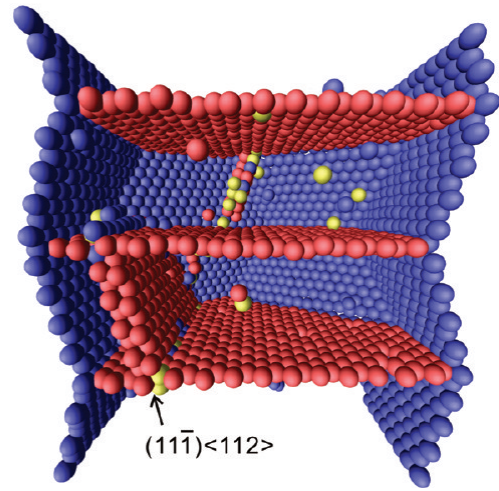

Figure 4. Snapshots of simulations showing the atomic details of nucleation and propagation of the very first dislocations in faceted Au nanowires at $300 \mathrm{~K}$ with (a) $D=20 \mathrm{~nm}$ and TBS $=8 \mathrm{~nm}$, and (b) $D=8 \mathrm{~nm}$ and TBS $=2 \mathrm{~nm}$. All atoms in perfect FCC arrangement have been removed for clarity.

limit. As such, it turns out that a competition between full $\{001\}\langle 110\rangle$ dislocation nucleation from the free surface and homogeneous plastic flow operates in faceted Au NWs as the critical resolved shear stress approaches the ideal strength of $\mathrm{Au}$. An accurate prediction of the transition point between these two mechanisms highly relies on how one can calculate the image forces imposed by CTBs. However, the curved nature of disloca- tions emitted from the free surface in Au NWs at yield point makes the quantitative predication of image forces from CTBs more complicated than that for straight dislocations as in the model of Chen et al. ${ }^{46}$

\section{CONCLUSIONS}

On the basis of classical MD simulations, we have shown a strong dependence of the tensile strength of Au NWs on both internal and external microstructures, which included randomly oriented polycrystalline NWs, bamboo-like NWs with nanoscale twins, perfectly circular NWs, and NWs with surface facets. The strongest microstructure was achieved in zigzag Au NWs made of a parallel arrangement of CTBs and $\{11 \overline{1}\}$ surface facets, whose strength is much larger than that of all perfectly circular Au NWs of identical size, regardless of their internal structure. The enhanced strength of faceted Au NWs can be interpreted from the surface morphology of $\{11 \overline{1}\}$ surface facets, which enforce all $\{11 \overline{1}\}$ slip planes to be parallel to a facet of the free surface. As a result, faceted Au NWs yield by a novel mechanism through site-specific emission of full $\{001\}\langle 110\rangle$ dislocations at the intersection of CTBs with the free surface, instead of the common $\{11 \overline{1}\}\langle 112\rangle$ partial slip observed in FCC metals. Furthermore, the yield strength of faceted Au NWs dramatically increases with decreasing twin boundary spacing. It is demonstrated that such phenomenon results from the effect of repulsive image forces imposed by CTBs on the glide of full dislocations. Most importantly, the yield strength of faceted Au NWs approaches the ideal strength in Au when the twin boundary spacing is decreased below a critical limit, accompanied by a transition in the yielding mechanism from surface dislocation nucleation to homogeneous dislocation nucleation. Our findings therefore reveal that microstructural design with surface faceting and coherent twin interfaces enables Au NWs to mechanically behave like infinite, defect-free crystals, whose elastic limit has classically set an upper bound for strength in crystalline materials. This study, which provides fundamental progress in nanoscale plasticity, will also stimulate new experimental studies in the areas of metal NW synthesis and nanostructure mechanics.

\section{METHODS}

MD Simulations. Nonequilibrium MD simulations were performed using LAMMPS ${ }^{47}$ molecular simulator with an embedded-atom-method potential for Au developed by Grochola et al. $^{48}$ This potential enables the simulations of realistic morphologies of nanoparticles in $\mathrm{Au}$, and accurately predicts the stacking fault and surface energies of this metal. ${ }^{48}$ Nanocrystalline Au NWs were created by standard Voronoi construction. ${ }^{49}$ To generate faceted NWs with desired size, a cylindrical twinned NW with a larger diameter was constructed, and six $\{11 \overline{1}\}$ facets were carved out on the surface of each grain by the removal of atoms beyond the $\{11 \overline{1}\}$ planes intersecting the free surface, similar to the surface morphology of semiconducting NWs observed experimentally. ${ }^{28-31}$ These NWs were oriented along the [111] crystallographic direction. Periodic boundary conditions were imposed in all NWs along the [111] axis, while the NWs were kept free in all other directions. For all NWs, the periodic length along the axis was equal to $32 \mathrm{~nm}$. The diameter of all cylindrical NWs was $20 \mathrm{~nm}$. The time step was $5 \mathrm{fs}$. Each model was relaxed for 100 ps prior to deformation under zero pressure using constant NPT integration with Nose-Hoover thermostat. The NW was deformed in tension by stretching the simulation box at a constant strain rate of 
$2.7 \times 10^{7} \mathrm{~s}^{-1}$ along the [111] direction with NVT integration The temperature was kept equal to $300 \pm 2 \mathrm{~K}$. The Virial theorem $^{50}$ was used to calculate the axial stress in the NWs at 10 ps intervals. The average atomic volume was calculated accurately in the cylindrical NW, where a negligible variation of less than $1 \%\left(1.678 \pm 0.015 \times 10^{-29} \mathrm{~m}^{3}\right)$ was observed during the tensile deformation. For simplicity, a constant atomic volume of $1.678 \times 10^{-29} \mathrm{~m}^{3}$ was assumed in all faceted NWs. The atomic structure of NWs during deformation was conducted at 10 ps intervals using Ackland and Jones' crystal structure analysis. ${ }^{51}$

Acknowledgment. The authors gratefully acknowledge support from the National Science Foundation (NSF) CAREER program (grant DMR-0747658) and the computational resources by the Vermont Advanced Computing Center (grant NASA NNX06AC88G).

Supporting Information Available: Movie showing the dynamic process of homogeneous dislocation nucleation. This material is available free of charge via the Internet at http://pubs. acs.org.

\section{REFERENCES AND NOTES}

1. Seeger, A. In Dislocations and Mechanical Properties of Crystals; Fisher, J. C., Johnston, W. G., Thomson, R., Eds.; John Wiley and Sons Inc.: New York, 1957; pp 243-329.

2. Hall, E. O. The Deformation and Ageing of Mild Steel. Proc. Phys. Soc. 1951, B64, 747-753.

3. Petch, N. J. The Cleavage Strength of Polycrystals. J. Iron Steel Inst. 1953, 174, 25-26.

4. Lu, L.; Shen, Y.; Chen, X.; Qian, L.; Lu, K. Ultrahigh Strength and High Electrical Conductivity in Copper. Science 2004, 304, 422-426.

5. Lu, L.; Chen, X.; Huang, X.; Lu, K. Revealing the Maximum Strength in Nanotwinned Copper. Science 2009, 323, 607610.

6. Lu, K.; Lu, L.; Suresh, S. Strengthening Materials by Engineering Coherent Internal Boundaries at the Nanoscale. Science 2009, 324, 349-352.

7. SchiØtz, J.; Jacobsen, K. W. A Maximum in the Strength of Nanocrystalline Copper. Science 2003, 301, 1357-1359.

8. Yamakov, V.; Wolf, D.; Phillpot, S. R.; Mukherjee, A. K.; Gleiter, H. Deformation-Mechanism Map for Nanocrystalline Metals by Molecular-Dynamics Simulation. Nat. Mater. 2004, 3, 43-47.

9. Uchic, M. D.; Dimiduk, D. M.; Florando, J. N.; Nix, W. D. Sample Dimensions Influence Strength and Crystal Plasticity. Science 2004, 305, 989.

10. Shan, Z. W.; Mishra, R. K.; Syed Asif, S. A.; Warren, O. L.; Minor, A. M. Mechanical Annealing and Source-Limited Deformation in Submicrometre-Diameter Ni Crystals. Nat. Mater. 2008, 7, 115-119.

11. Buzzi, S.; Dietiker, M.; Kunze, K.; Spolenak, R.; LÖffler, J. F. Deformation Behavior of Silver Submicrometer-Pillars Prepared by Nanoimprinting. Philos. Mag. 2009, 89, 869884.

12. Gall, K.; Diao, J.; Dunn, M. L. The Strength of Gold Nanowires. Nano Lett. 2004, 4, 2431-2436.

13. Wu, B.; Heidelberg, A.; Boland, J. J. Mechanical Properties of Ultrahigh-Strength Gold Nanowires. Nat. Mater. 2005, 4 525-529.

14. Greer, J. R.; Nix, W. D. Nanoscale Gold Pillars Strengthened through Dislocation Starvation. Phys. Rev. B 2006, 73 , 245410.

15. Volkert, C. A.; Lilleodden, E. T. Size Effects in the Deformation of Submicron Au Columns. Philos. Mag. 2006 86, 5567-5579.

16. Maaß, R.; Petegem, S. V.; Swygenhoven, H. V.; Derlet, P. M. Time-Resolved Laue Diffraction of Deformation Micropillars. Phys. Rev. Lett. 2007, 99, 145505.

17. Maaß, R.; Petegem, S. V.; Zimmermann, J.; Borca, C. N.; Swygenhoven, H. V. On the Initial Microstructure of Metallic Micropillars. Scr. Mater. 2008, 59, 471-474.
18. Tian, M.; Wang, J.; Kurtz, J.; Mallouk, T. E.; Chan, M. H. W Electrochemical Growth of Single-Crystal Metal Nanowires via a Two-Dimensional Nucleation and Growth Mechanism. Nano Lett. 2003, 3, 919-923.

19. Wang, J.; Tian, M.; Mallouk, T. E.; Chan, M. H. W. Microtwinning in Template-Synthesized Single-Crystal Metal Nanowires. J. Phys. Chem. B 2004, 108, 841-845.

20. Wang, C.; Hu, Y.; Lieber, C. M.; Sun, S. Ultrathin Au Nanowires and Their Transport Properties. J. Am. Chem. Soc. 2008, 130, 8902-8903.

21. Huo, Z.; Tsung, C.; Huang, W.; Zhang, X.; Yang, P. Sub-Two Nanometer Single Crystal Au Nanowires. Nano Lett. 2008, 8, 2041-2044.

22. Halder, A.; Ravishankar, N. Ultrafine Single-Crystalline Gold Nanowire Arrays by Oriented Attachment. Adv. Mater. 2007, 19, 1854-1858.

23. Kiely, C. J.; Fink, J.; Brust, M.; Bethell, D.; Schiffrin, D. J. Spontaneous Ordering of Bimodal Ensembles of Nanoscopic Gold Clusters. Nature 1998, 396, 444-446.

24. Lofton, C.; Sigmund, W. Mechanisms Controlling Crystal Habits of Gold and Silver Colloids. Adv. Funct. Mater. 2005, 15, 1197-1208.

25. Shen, M.; Du, Y.; Yang, P.; Jiang, L. Morphology Control of the Fabricated Hydrophobic Gold Nanostructures in W/O Microemulsion under Microwave Irradiation. J. Phys. Chem. Solid. 2005, 66, 1628-1634.

26. Elechiguerra, J. L.; Reyes-Gasga, J.; Yacaman, M. J. The Role of Twinning in Shape Evolution of Anisotropic Noble Metal Nanostructures. J. Mater. Chem. 2006, 16 3906-3919.

27. Wang, J.; Huang, H.; Kesapragada, S. V.; Gall, D. Growth of Y-Shaped Nanorods through Physical Vapor Deposition. Nano Lett. 2005, 5, 2505-2508.

28. Banerjee, R.; Bhattacharya, A.; Genc, A.; Arora, B. M. Structure of Twins in GaAs Nanowires Grown by the Vapour-Liquid-Solid Process. Philos. Mag. Lett. 2006, 86, 807-816.

29. Algra, R. E.; Verheijen, M. A.; BorgstrÖm, M. T.; Feiner, L. F.; Immink, G.; Enckevort, W. J. P. V.; Vlieg, E.; Bakkers, E. P. A. M. Twinning Superlattices in Indium Phosphide Nanowires. Nature 2008, 456, 369-372.

30. Arbiol, J.; Morral, A. F. I.; Estradé, S.; Peiró, F.; Kalache, B.; Cabarrocas, P. R. I.; Morante, J. R. Influence of the (111) Twinning on the Formation of Diamond Cubic/Diamand Hexagonal Heterostructures in Cu-Catalyzed Si Nanowires. J. Appl. Phys. 2008, 104, 064312.

31. Caroff, P.; Dick, K. A.; Johansson, J.; Messing, M. E.; Deppert, K.; Samuelson, L. Controlled Polytypic and Twin-Plane Superlattices in III-V Nanowires. Nat. Nanotechnol. 2009, 4, 50-55.

32. Shim, H. W.; Zhang, Y.; Huang, H. Twin Formation during SiC Nanowire Synthesis. J. Appl. Phys. 2008, 104, 063511.

33. Monk, J.; Farkas, D. Tension-Compression Asymmetry and Size Effects in Nanocrystalline Ni Nanowires. Philos. Mag. 2007, 87, 2233-2244.

34. Cao, A.; Wei, Y.; Ma, E. Grain Boundary Effects on Plastic Deformation and Fracture Mechanisms in Cu Nanowires: Molecular Dynamics Simulations. Phys. Rev. B 2008, 77, 195429.

35. Afanasyev, K. A.; Sansoz, F. Strengthening in Gold Nanopillars with Nanoscale Twins. Nano Lett. 2007, 7, 2056-2062

36. Deng, C.; Sansoz, F. Enabling Ultrahigh Plastic Flow and Work Hardening in Twinned Gold Nanowires. Nano Lett. 2009, 9, 1517-1522.

37. Zhang, Y.; Huang, H. Do Twin Boundaries Always Strengthen Metal Nanowires. Nano. Res. Lett. 2008, 4, 34-38.

38. Leach, A. M.; McDowell, M.; Gall, K. Deformation of TopDown and Bottom-Up Silver Nanowires. Adv. Func. Mater. 2006, 17, 43-53.

39. Ji, C.; Park, H. S. The Coupled Effects of Geometry and Surface Orientation on the Mechanical Properties of Metal Nanowires. Nanotechnology 2007, 18, 305704 
40. Hyde, B.; Espinosa, H. D.; Farkas, D. An Atomistic Investigation of Elastic and Plastic Properties of $A u$ Nanowires. J. Miner., Met., Mater. Soc. 2005, 57, 62-66.

41. FrØseth, A.; Swygenhoven, H. V.; Derlet, P. M. The Influence of Twins on the Mechanical Properties of nc-Al. Acta Mater. 2004, 52, 2259-2268.

42. Deng, C.; Sansoz, F. Size-Dependent Yield Stress in Twinned Gold Nanowires Mediated by Site-Specific Surface Dislocation Emission. Appl. Phys. Lett. 2009, 95, 091914.

43. When deformed in tension along the [111] crystal orientation, bulk Au specimens yielded by homogeneous nucleation of a $\{111\}\langle 112\rangle$ partial dislocation with a Schmid factor equal to 0.314 , and therefore the ideal shear stress for this slip corresponds to $1.73 \mathrm{GPa}$. This value agrees very well with $1.78 \mathrm{GPa}$, the prediction by Diao et al. ${ }^{12}$ from MD by directly shearing of two $\mathrm{Au}\{11 \overline{1}\}$ planes in $\langle 112\rangle$ direction. It should also be mentioned that the ideal strength obtained from classical atomistic simulations is higher than $1.42 \mathrm{GPa}$, the value calculated by first-principle calculations for this slip systems in $\mathrm{Au}^{52}$ The deviation may originate from the embedded-atom method (EAM) Au potential ${ }^{47}$ used in this study.

44. Diao, J.; Gall, K.; Dunn, M. L. Yield Strength Asymmetry in Metal Nanowires. Nano Lett. 2004, 4, 1863-1867.

45. Wang, J.; Huang, H. Novel Deformation Mechanism of Twinned Nanowires. Appl. Phys. Lett. 2006, 88, 203112.

46. Chen, Z.; Jin, Z.; Gao, H. Repulsive Force between Screw Dislocation and Coherent Twin Boundary in Aluminum and Copper. Phys. Rev. B 2007, 75, 212104.

47. Plimpton, S. J. J. Comp. Phys. 1995, 117, 1. (http://lammps.sandia.gov).

48. Grochola, G.; Russo, S. P.; Snook, I. K. On Fitting a Gold Embedded Atom Method Potential Using the Force Matching Method. J. Chem. Phys. 2005, 123, 204719.

49. Voronoi, G. Recherches sur les Paralléloèdres Primitives. J. Reine. Angew. Math. 1908, 134, 198-287.

50. Diao, J.; Gall, K.; Dunn, M. L. Atomistic Simulation of the Structure and Elastic Properties of Gold Nanowires. J. Mech. Phys. Solids 2004, 52, 1935-1962.

51. Ackland, G. J.; Jones, A. P. Applications of Local Crystal Structure Measures in Experiment and Simulation. Phys. Rev. B 2006, 73, 054104

52. Ogata, S.; Li, J.; Hirosaki, N.; Shibutani, Y.; Yip, S. Ideal Shear Strain of Metals and Ceramics. Phys. Rev. B 2004, 70, 104104. 